\title{
Le patrimoine au service du commerce? Une lecture sémiologique des rues commerçantes du Marais et du Vieux Lille
}

Retailers using heritage: a semiologic reading of the Marais et Vieux Lille main

shopping streets

Wenn der Handel das Erbe benutzt: eine semiotische Lektüre der

Einkaufsstrassen des Marais und Vieux Lille.

Anne-Cécile Mermet

\section{OpenEdition}

\section{Journals}

Electronic version

URL: http://journals.openedition.org/rge/5087

DOI: $10.4000 /$ rge.5087

ISSN: 2108-6478

\section{Publisher}

Association des géographes de l'Est

Printed version

Date of publication: 30 December 2013

ISSN: 0035-3213

\section{Electronic reference}

Anne-Cécile Mermet, « Le patrimoine au service du commerce? Une lecture sémiologique des rues commerçantes du Marais et du Vieux Lille », Revue Géographique de l'Est [Online], vol. 53 / 3-4 | 2013, Online since 06 July 2014, connection on 08 September 2020. URL : http://journals.openedition.org/ rge/5087 ; DOI : https://doi.org/10.4000/rge.5087

This text was automatically generated on 8 September 2020

Tous droits réservés 


\title{
Le patrimoine au service du commerce? Une lecture sémiologique des rues commerçantes du Marais et du Vieux Lille
}

\author{
Retailers using heritage: a semiologic reading of the Marais et Vieux Lille main \\ shopping streets \\ Wenn der Handel das Erbe benutzt: eine semiotische Lektüre der \\ Einkaufsstrassen des Marais und Vieux Lille.
}

\section{Anne-Cécile Mermet}

\section{Introduction}

1 On peut définir dans une perspective constructiviste la patrimonialisation comme un processus social se traduisant dans un premier temps par l'attribution d'une valeur symbolique à une trace du passé et dans un deuxième temps par le marquage matériel de cette trace (Veschambre, 2008). En cela, les centres historiques apparaissent comme une presque « évidence patrimoniale ». En France, ce sont en effet des quartiers qui ont été le plus tôt reconnus et marqués comme patrimoine. Fers de lance de la mise en place des monuments historiques puis des secteurs sauvegardés, les centres historiques ont acquis une indéniable fonction patrimoniale, venant s'ajouter à la multiplicité classique des fonctions urbaines dans ces quartiers, parmi lesquelles la fonction commerçante, aujourd'hui en pleine renaissance et restructuration.

2 La requalification des centres historiques peut donc être lue comme une forme de marquage patrimonial, rendant visible la valeur (morale, symbolique, économique, sociale) de ces lieux. La patrimonialisation de ces quartiers n'est donc pas neutre en 
termes de valorisation de l'espace urbain. Parmi la diversité des acteurs intervenant dans la production (au sens d'Henri Lefebvre, 2000 [1974]) de ces quartiers, cet article analyse la façon dont les commerçants instrumentalisent cette fonction patrimoniale pour participer à produire un espace de consommation original, principalement fondé sur la valorisation matérielle, symbolique et in fine économique de ce cadre patrimonial.

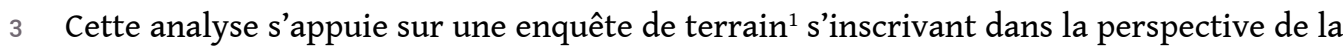
définition structuraliste que Jean Baudrillard donne à la consommation (1970), fondée sur la notion de signe, et dans celle des travaux de Pierre Boudon sur la sémiologie spatiale, fondée sur « une construction méthodologique associant formes de l'urbain [...] et discours sur l'urbain» (Boudon, 1977, p. 2). L'observation de deux centres historiques - le Marais et le Vieux Lille - et notamment, au niveau micro-géographique, de leurs boutiques, a donc été complétée par le recueil de discours (via des entretiens mais aussi des sources secondaires) sur ces commerces.

\section{Terrains et méthodologie}

4 Bien que situé dans des contextes métropolitains différents (ville globale/ville en cours de métropolisation), le Marais et le Vieux Lille connaissent depuis quelques décennies des dynamiques urbaines très comparables. Il s'agit d'une part de deux secteurs sauvegardés promulgués dans les années 1960, ayant bénéficié d'une politique de réhabilitation ayant mis en avant un patrimoine bâti majoritairement hérité du xviiie siècle dans les deux cas, et s'étant traduite également par une forte dynamique de gentrification assez précoce. Ces deux quartiers ont également connu de profondes mutations commerciales sensiblement comparables (baisse du commerce alimentaire et de l'équipement de la maison à la faveur essentiellement du prêt-à-porter, des agences et des galeries). En outre, certaines rues de ces quartiers (rue des Rosiers, rue des Francs-Bourgeois pour le Marais ; rue de la Grande Chaussée, rue de la Monnaie pour le Vieux Lille) connaissent un ample mouvement de «franchisation » de leurs boutiques, et figurent régulièrement dans le «top ten » des valeurs locatives parisiennes (pour le Marais) et provinciales (pour le Vieux Lille) établi par l'Argus de l'enseigne.

5 L'analyse s'appuie sur l'observation de ces deux centres historiques ${ }^{2}$, et notamment, au niveau micro-géographique, de leurs boutiques, ainsi que sur une campagne d'entretiens conduite auprès d'environ 80 acteurs locaux (commerçants, acteurs associatifs et institutionnels). Sur 61 entretiens réalisés avec des commerçants, 10 ont été conduits auprès de développeurs ou de directeurs généraux d'enseignes (essentiellement dans le prêt-à-porter) sélectionnées parce qu'elles privilégient les centres-villes ou centres historiques dans leurs stratégies de localisation, 31 auprès de commerçants du Marais (24 indépendants et 7 franchisés ou succursalistes ${ }^{3}$ ) et 20 auprès de commerçants du Vieux Lille $(15$ indépendants, 5 franchisés ou succursalistes). Ces discours ont été complétés par la constitution d'un corpus de sources secondaires (presse, sites Internet). L'objectif global de ce dispositif était de décrypter le sens et les valeurs que les commerçants donnent à ce cadre matériel historique par la mise en articulation des signes du commerce (devanture, vitrine, enseigne, mobilier...) et des signes du patrimoine (caractéristiques physiques des cellules commerciales en centre historique, présence d'éléments historiques...). 
Figure 1 : Localisation des entretiens réalisés avec les commerçants du Marais (gauche) et du Vieux Lille (droite)

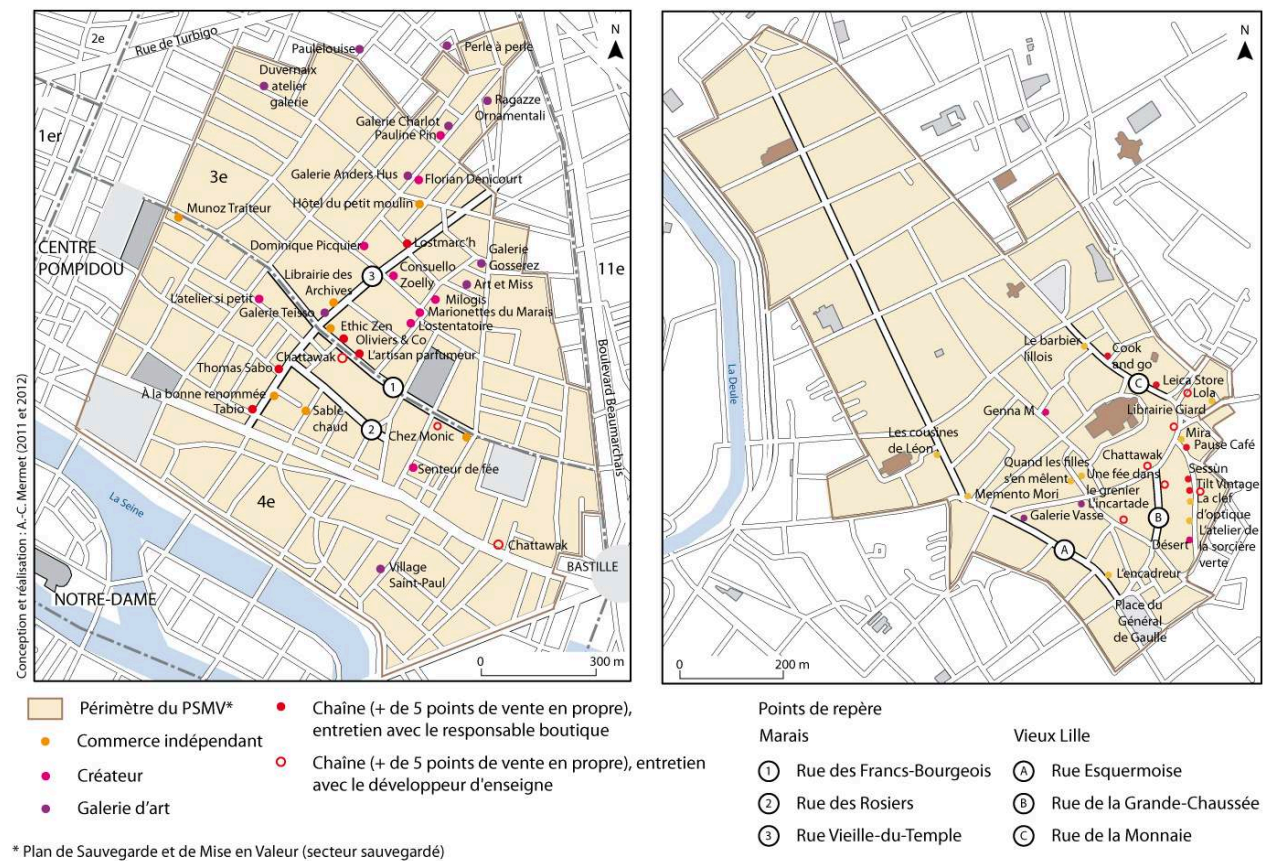

On se penchera tout d'abord sur l'esthétique particulière que produit l'articulation volontaire, par les commerçants, entre les signes du patrimoine et du commerce, avant, de tenter de proposer une grille de lecture symbolique permettant de décoder le discours visuel ainsi produit.

\section{Le décor patrimonial, une ressource esthétique pour les commerçants}

7 La tendance actuelle du merchandising ${ }^{4}$ tend à donner presque autant d'importance au cadre spatial dans lequel le produit est vendu qu'au produit lui-même (Sackrider, 2008). À l'heure où le milieu du marketing utilise de plus en plus les apports de la psychologie environnementale (Rieunier, 2006), l'esthétique visuelle des boutiques et de leur contexte urbain, joue donc un rôle majeur dans les stratégies de vente des commerçants. Choisir une localisation en centre historique, c'est donc s'inscrire dans un cadre matériel truffé de signes patrimoniaux marqués ou en attente de marquage, mais dont la valeur, d'abord esthétique, est unanimement reconnue. La présence de ces signes, permise par la localisation en centre historique, constitue ainsi une stratégie de choix dans cette nécessaire production d'un environnement de consommation qualitatif, comme l'ont confirmé l'observation des cellules commerciales ainsi que les entretiens et l'analyse du corpus de sources secondaires. De fait, l'esthétique des boutiques de centre historique réserve une place de choix aux éléments hérités du passé des lieux, qu'il s'agisse de la valorisation d'éléments patrimoniaux « déjà là » ou de l'invention de nouveaux signes patrimoniaux dans le ton de cette esthétique historicisante. 


\section{A. Utiliser et valoriser le décor patrimonial déjà là}

8 La première stratégie qui émane des entretiens et des séances d'observation consiste en une valorisation par les commerçants, du " décor patrimonial déjà là ", mais en attente de marquage. Les locaux commerciaux du Marais ou du Vieux Lille détiennent en effet généralement des signes du patrimoine - plus ou moins visibles, dans un plus ou moins bon état - qu'il s'agisse du bâtiment dans son ensemble, de la devanture ou de l'intérieur de la boutique. Les commerçants composent en effet volontiers avec ces éléments signifiants, indépendamment des contraintes liées aux servitudes patrimoniales. Lors des entretiens, la plupart des commerçants a affirmé avoir choisi de réhabiliter un élément témoin de l'épaisseur historique de leur boutique, certains ayant même parfois regretté l'absence de tels signes dans leur local. Cette réhabilitation volontaire d'éléments patrimoniaux, destinée à « marquer » ce patrimoine, s'incarne le plus fréquemment dans la restauration ou dans la simple mise en valeur visuelle d'éléments appartenant à la structure du bâtiment : mur en pierres (pour le Marais) ou en briques (pour le Vieux Lille) apparentes, vieilles poutres, éléments particulièrement originaux de la devanture etc. À titre d'exemple, la boutique de prêt-à-porter Sessùn ${ }^{5}$ (cf. figure 3c), installée depuis début 2010 dans la rue de la Clef (Vieux Lille) à la place d'un multimarque de prêt-à-porter qui avait notamment choisi de « lisser » l'allure du local en installant du placo-plâtre sur les murs, a, lors des travaux, eu la «bonne surprise » (sic) de découvrir un mur en briques « exploitable », que la marque a choisi de restaurer (nettoyage principalement) et de mettre en avant dans la décoration de son point de vente.

9 De façon plus radicale, les commerçants peuvent choisir d'eux-mêmes de réhabiliter certains éléments patrimoniaux jusqu'alors inconnus et dont ils sont les « découvreurs » à l'occasion de leurs travaux d'installation. C'est par exemple le cas du restaurant-boucherie-traiteur Le Barbier lillois ${ }^{6}$, situé rue de la Monnaie au cœur du Vieux Lille. Lors des importants travaux de réhabilitation du bâtiment qui tombait en ruine, le propriétaire a découvert la présence de traces de plats à barbe peints sur les arcs de gré de la devanture (cf. figure 2a). Il a alors engagé une recherche historique aux Archives pour retracer l'histoire de cette boutique, à l'issue de laquelle il a pu conclure que ce local commercial abritait au début du xviiie siècle un maîtreperruquier. Il a dès lors pris le parti de restaurer, avec la collaboration du STAP ${ }^{7}$, ces peintures et de fonder l'intégralité de l'identité de sa boucherie - qui s'appelle significativement «Le barbier lillois » - sur cette histoire. On aurait pu également développer l'exemple de la Librairie des Archives dans le Marais (rue Vieille-du-Temple - figure 3a) dont le propriétaire a mis à jour, sous l'enseigne de son prédécesseur, une enseigne datant des années 1940, héritage du passé textile du quartier, qu'il a choisi de valoriser au détriment de la visibilité de sa propre enseigne. 
Figure 2 : Le Barbier lillois
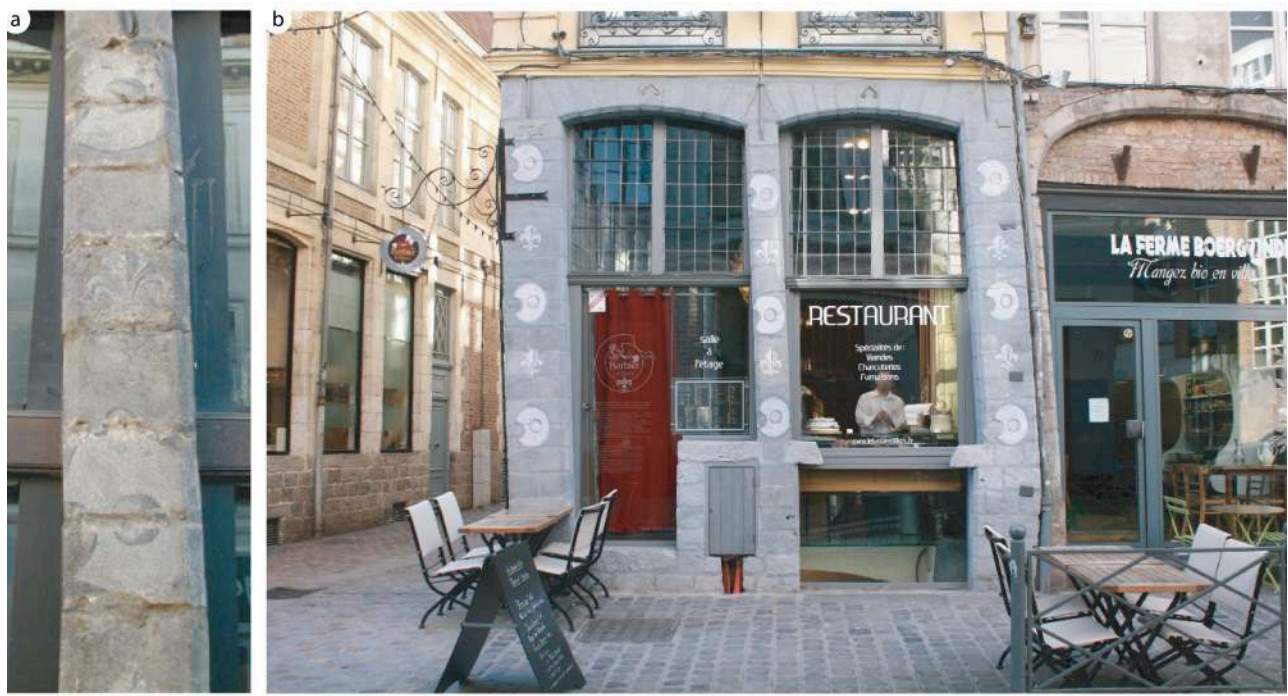

(a) : Les traces de peinture de plats à barbe sur les arcs de gré avant la restauration. Source Renaissance du Lille ancien ; (b) : La devanture après restauration. Source : A.-C. Mermet (janvier 2012)

Figure 3 : Exemples d'éléments patrimoniaux réhabilités par des commerçants
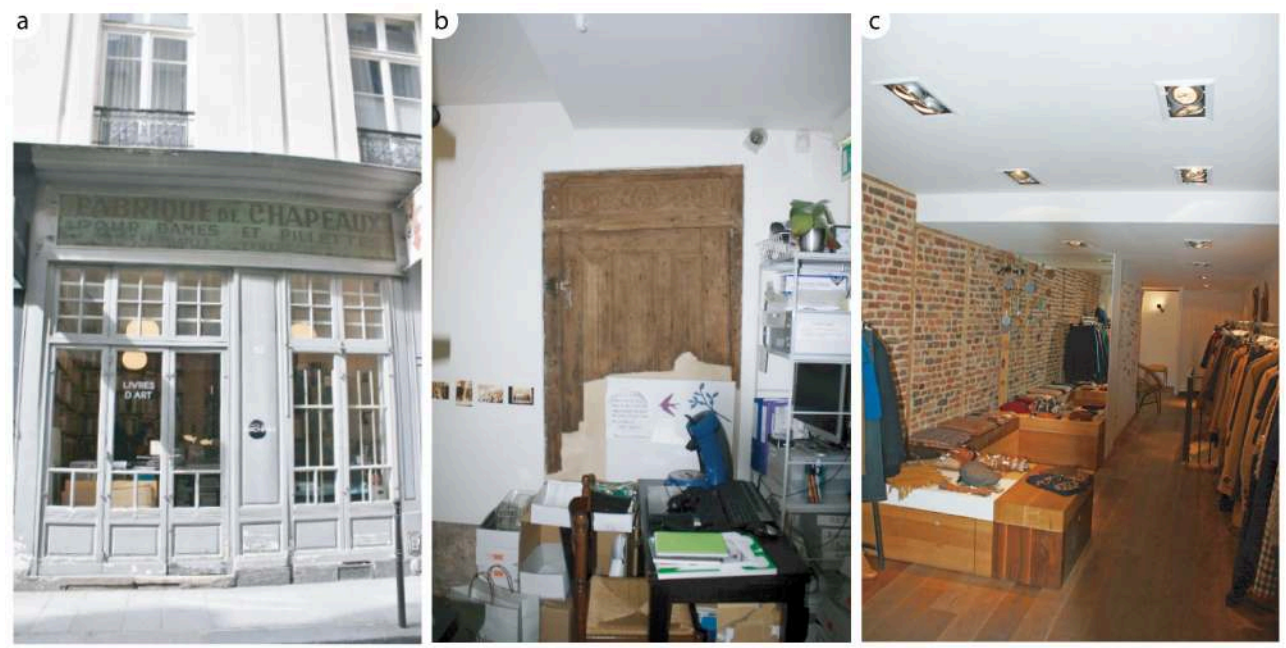

(a) : Librairie des Archives, 83 rue Vieille-du-Temple, Marais (indépendant). Source : A.C. Mermet (mai 2011) ; (b) : Tabio, 15 rue Vieille-du-Temple, Marais (succursale). Source : A.-C. Mermet (avril 2011);

(c) : Sessùn, 35 rue de la Clef, Vieux Lille (succursale). Source : A.-C. Mermet (novembre 2011).

La réhabilitation et la valorisation du cadre patrimonial par les commerçants concernent donc aussi bien la devanture (3a: l'enseigne «Fabrique de chapeaux pour dames et fillettes » est bien plus visible que la minuscule vitrophanie "Librairie d'art " apposée sur la vitrine au dessus de la porte d'entrée) que l'intérieur des commerces ( $3 \mathrm{~b}$ : la porte du xvie siècle a été découverte par les responsables de la boutique de chaussettes Tabio et restaurée à leur initiative). Les commerçants apparaissent donc très clairement comme des acteurs de la réhabilitation et du marquage du patrimoine en centre historique. Si l'objectif principal n'est généralement pas ici le «bien commun » mais l'instrumentalisation du décor patrimonial à des fins de marketing, il 
n'en reste pas moins qu'il s'agit là d'un marquage ayant une visibilité particulièrement forte pour des espaces ouverts au public.

Ces illustrations montrent par ailleurs que ce constat est valable pour les commerçants indépendants (Le barbier lillois, la Librairie des Archives) mais aussi pour les chaînes (Tabio, Sessùn) qui, bien qu'elles doivent dans la plupart des cas respecter des " concepts» de boutique dans leur aménagement intérieur, s'accommodent très bien des spécificités patrimoniales des locaux qu'elles perçoivent comme une plus-value en termes de merchandising, comme l'illustre le cas de Chattawak. Marque de prêt-àporter française moyen-haut de gamme fondée en 1987 en plein développement, cette chaîne qui privilégie presque exclusivement les localisations en centre-ville (90\% de ses boutiques ${ }^{8}$ ) a clairement tendance à rechercher des locaux atypiques dans la mesure où cela lui permet cependant de s'exprimer et de s'implanter rapidement, comme l'explique la responsable développement de l'enseigne :

- Est-ce que pour certaines boutiques, vous avez dû adapter votre concept à la configuration de la boutique, ou est-ce que si, par exemple, il y a des éléments patrimoniaux comme des vieilles poutres ou un mur en pierres apparentes, vous essayez de les conserver?

- Oui. On essaie de garder ce qu'il y a. On s'adapte à l'environnement du magasin. Par exemple, si vous allez à Lille, vous verrez qu'au milieu du magasin, il y a un poteau en pierre, il $y$ a des briques apparentes, ça, on n'y a pas touché parce que c'est très joli et ce serait dommage d'y toucher. On a travaillé avec ce qui existait déjà dans le magasin. Bon, 9 fois sur 10, les magasins sont pas forcément en bon état et il faut refaire ça, mais par exemple le magasin de Lille, c'est vrai qu'il avait un caractère, de par sa décoration, avec les briques apparentes et tout ça, donc ça on l'a conservé [...]. Si ça nous empêche pas de nous exprimer, je pense que c'est un vrai atout de valoriser l'architecture de la boutique si c'est un peu atypique. Ça permet aussi de ne pas offrir une boîte et quelque chose de trop lisse, trop propre, qui peut être effrayant parfois et qui peut aussi avoir un côté trop froid qu'on cherche pas à avoir. Donc des poutres, je pense que c'est chouette aussi parce que justement il y a un côté plus chaleureux... qu'on cherche pas à effacer.

12 Les valeurs véhiculées par le nouveau concept des boutiques Chattawak (le côté chaleureux, urbain, raffiné) sont donc renforcées par la présence visible des signes du patrimoine (la vieille poutre et les briques apparentes de la boutique de Lille, la « vieille façade » de celle de Strasbourg, «les colombages» de celle de Rouen...) et entrent véritablement en cohérence avec celui-ci. Au regard des entretiens conduits avec les développeurs d'enseignes et les responsables de boutiques intégrées à des réseaux, ce type de stratégie ne semble pas être l'exception pour des chaînes moyen-haut de gamme privilégiant des localisations centrales et tentant de véhiculer une image de prestige.

\section{B. Accentuer les signes du patrimoine}

Cette volonté de bénéficier d'un cadre patrimonial incite dans de nombreux cas les commerçants à accentuer les signes du patrimoine voire à en inventer de nouveaux afin de donner un surplus d'historicité à leur point de vente.

En ce qui concerne la devanture, l'ajout de nouveaux signes patrimoniaux passe souvent par le recours à une typographie à forte connotation passéiste (italique, style manuscrit...). Mais c'est surtout à travers l'aménagement intérieur des boutiques que 
cette tendance est sensible. Cela passe, tout d'abord, par l'utilisation très fréquente de mobilier chiné, déniché chez des brocanteurs. Si certaines chaines (Chattawak, Catimini, Stéphane Gontard, Zapa, etc.) ont développé leur propre mobilier que l'on retrouve d'une boutique à une autre, du fait de la nécessité de respect d'un " concept » véhiculant l'identité de la marque (avec toutefois quelques petites adaptations), d'autres chaînes (Tabio, Sessùn, etc.), et la plupart des commerçants indépendants enquêtés, ont recours à un mobilier trouvé dans des brocantes. Il s'agit parfois simplement de faire des économies - mais pas toujours - et, y compris dans ce cas, le choix de ce type d'ameublement est destiné à renforcer l'identité patrimoniale des locaux investis. On peut par exemple citer le concept-store indépendant Memento Mori, situé dans un rez-de-chaussée commercial d'un bâtiment à forte connotation historique rue Esquermoise dans le Vieux Lille. Cette boutique diffuse des produits très contemporains en lien avec la thématique gothique, mais avait, dès le départ, pris le parti de ne pas proposer "une boutique design, épurée » en mettant en avant le fait d'avoir investi « un lieu qui a du cachet, une histoire ». L'aménagement intérieur joue donc un grand rôle dans l'affirmation de cette identité, comme l'explique la cofondatrice de la boutique :

- Et donc là, les meubles, c'est un mixte de tables de drapier qu'on a achetées, découpées, et qui sont coupées en deux pour faire des tablettes, sur les côtés. C'est pas des tables d'occasion, par contre tout le reste du mobilier, les petites vitrines, les grandes armoires et tout, c'est des meubles qu'on a chinés, et qui ont été décapés et repeints. Les tables et tout... tout ça aussi [...]

- Vous avez récupéré ça chez des brocanteurs?

- Oui, on travaille avec des brocanteurs, des antiquaires... c'est le troc de Lille... c'est un magasin de dépôt-vente en fait, où les gens revendent tous leurs meubles de grand-mère, tous leurs trucs, c'est une espèce d'immense entrepôt [...] On est toujours dans une histoire d'univers.

De façon encore plus significative, le recours à un tel type de mobilier, ou à l'installation d'un parquet "authentiquement ancien » acheté chez des récupérateurs se fournissant dans d'anciens châteaux ou appartements haussmanniens, est parfois explicitement destiné à compenser l'absence de signes du patrimoine visibles dans la configuration du local commercial (Senteurs de fée dans le Marais, Genna M. dans le Vieux Lille, infra).

Cette « invention » de nouveaux signes du patrimoine est parfois plus lourde en allant jusqu'à la recréation ex nihilo d'éléments patrimoniaux dont on aurait aimé pouvoir disposer dans la décoration de la boutique. C'est par exemple le cas de la boutique Oliviers \& Co de la rue des Francs-Bourgeois ${ }^{9}$ (Marais) qui a reconstitué un mur en pierres apparentes dans son local commercial.

\section{Faire voir le patrimoine}

17 L'objectif global de ces stratégies de marquage patrimonial par les commerçants n'est pas complètement dénué de visées marketing et économiques. Sur le plan visuel, il s'agit de proposer au consommateur d'entrer non pas dans une boutique lambda et stéréotypée comme pourrait l'être une boutique de centre commercial, mais de faire entrer le chaland dans un objet patrimonial unique et de le lui montrer. Dans cette volonté de mettre en avant ce statut exceptionnel de l'allure de la boutique, il faut 
souligner le rôle majeur de la vitrine et des jeux de transparence qu'elle permet (Péron, 1993), en offrant au regard du chaland qui déambule dans la rue les caractéristiques patrimoniales de leur point de vente.

Figure 4 : Jeux de transparence : entretenir l'attractivité de la boutique, objet patrimonial unique, grâce aux vitrines

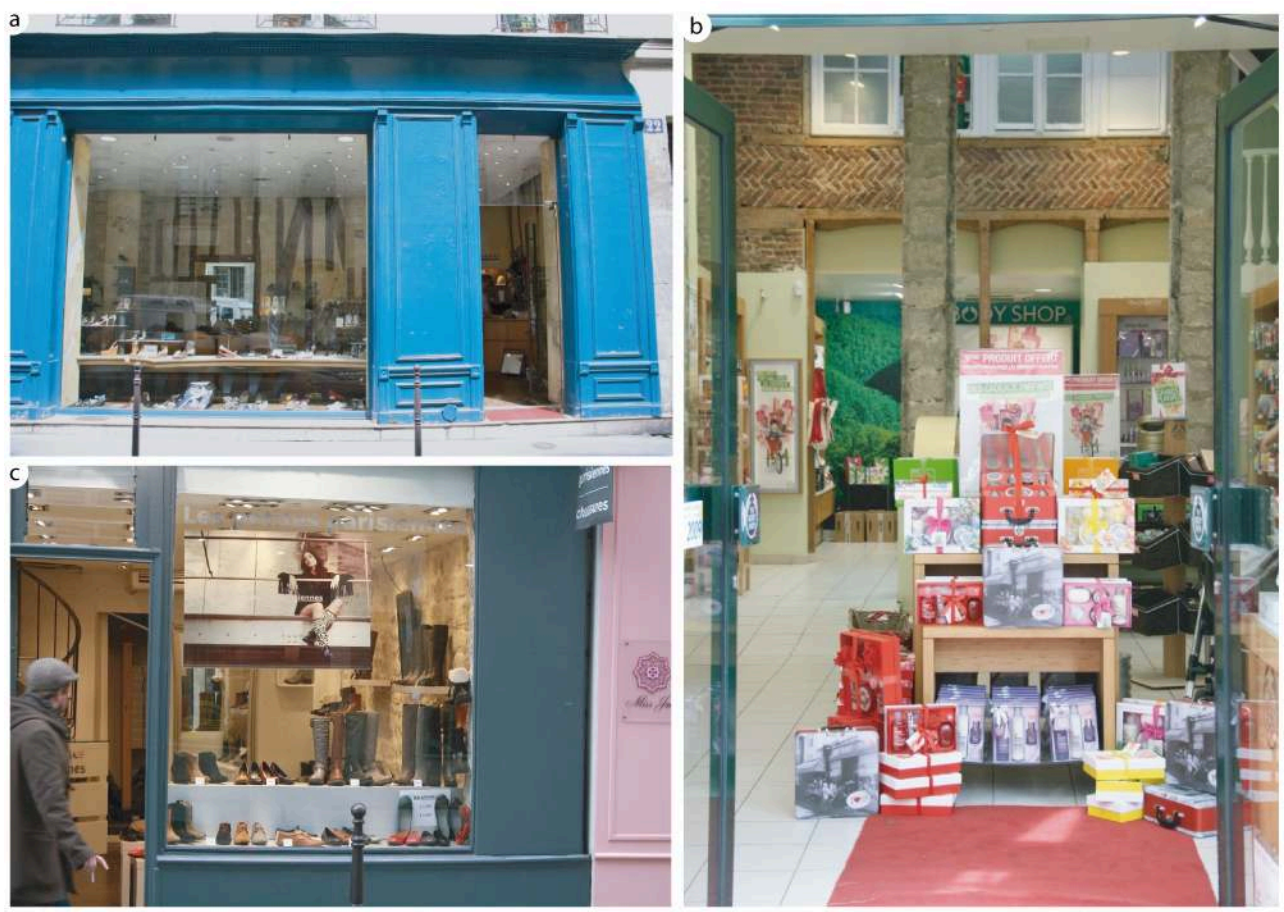

(a) Boutique de chaussures Jean-Claude Monderer, 22 rue des Francs-Bourgeois, Marais (succursale). Source : A.-C. Mermet (mars 2012) ; (b) Boutique The body shop, 6 rue Lepelletier, Vieux Lille

(succursale). Source : A.-C. Mermet (mai 2012); (c) Boutique de chaussures Les petites parisiennes, 32 rue de Sévigné, Marais (succursale). Source : A.-C. Mermet (mars 2012).

Ces clichés ont tous été pris depuis la rue et font clairement apparaître une stratégie de «mise en vue» des signes du patrimoine de ces points de vente, qu'il s'agisse de l'ensemble de la structure de la boutique (comme dans le cas de The Body Shop dans le Vieux Lille où l'ensemble de la façade du deuxième corps de bâtiment a été restauré et mis en valeur, les portes de la boutique sont ouvertes pour donner à voir l'écrin unique des produits vendus), d'éléments du décor intérieur (poutres (a), murs en pierre (c)).

Les signes du patrimoine jouent un rôle majeur dans la "théâtralisation » (Rieunier, 2006, p. 23) du point de vente en centre historique. Cette articulation entre les signes du commerce et les signes du patrimoine produit donc un espace de consommation à l'esthétique particulièrement soignée et qualitative.

\section{Dimension symbolique de cette nouvelle esthétique : vers une sémiologie spatiale d'une localisation en centre historique}

20 Cette description de la production esthétique des boutiques en centres historiques n'épuise pas l'analyse de l'articulation entre les signes du commerce et du patrimoine. Les entretiens ont en effet mis en évidence l'importance du registre du symbolique 
dans cette grammaire commune. Il s'agit donc de compléter l'analyse en s'intéressant au registre du symbolique tel que défini par Gilles Deleuze (1967), comme un registre médiateur entre le registre du réel ( $\mathrm{du}$ sensible et du matériel) et celui de l'imaginaire (de l'idéel et de l'intelligible) associant aux formes matérielles visibles des idées et des valeurs. On postulera ainsi que le marquage voire l'accentuation des signes $d u$ patrimoine par les commerçants participe d'une stratégie de mise en résonnance de la symbolique qualitative associée au patrimoine avec celle des objets vendus et de la marque défendue, afin de construire une "synergie lieu/produit» (Sackrider et al., 2008, p. 146). Dès lors, quelles sont les valeurs symboliques qui sont mises en exergue et pourquoi ? Il s'agit ici de mettre à jour la sémiologie spatiale mise en place dans ces boutiques, en s'appuyant sur les discours des acteurs commerçants commentant l'aménagement de leur point de vente et expliquant les raisons qui ont motivé de tels choix afin de produire une grille de lecture symbolique de ces nouveaux espaces de consommation. L'ensemble des entretiens réalisés avec les acteurs commerçants permet de dégager trois grandes valeurs liées au patrimoine bâti des centres historiques qui tendent à être instrumentalisées par les boutiques et produisent prestige et distinction : l'unicité, la continuité, et l'intimité/proximité.

\section{A. Unicité}

21 La synergie lieu patrimonial/boutique/produit s'exprime tout d'abord à travers l'idée d'unicité. L'un des objectifs majeurs de ces boutiques situées dans des quartiers hautement commerçants réside dans la nécessité de se différencier de la concurrence, et donc dans le besoin de mettre en valeur des éléments uniques, qu'il s'agisse des produits ou du cadre dans lequel ceux-ci sont présentés. Or le cadre patrimonial est par définition unique, quelle que soit l'échelle envisagée (quartier, rue, local commercial). Dès lors, l'une des hypothèses qui peut être émise pour expliquer cette synergie lieu/ produit réside dans la symbolique d'unicité associée au patrimoine qui pourrait constituer un critère majeur de différenciation dans ce type d'environnement très concurrentiel. On peut reprendre à ce sujet la réflexion développée par David Harvey autour de la notion de rente de monopole (2008, p. 24). Partant du postulat selon lequel le capitalisme est fondé sur le contrôle de rentes de monopole, c'est-à-dire sur le contrôle exclusif, par des acteurs sociaux, d'un objet qui doit être unique et non reproductible, il apparaît que les objets que l'on qualifie de "culturels ", et notamment les objets patrimoniaux, constituent de belles opportunités pour ce type de rente dans la mesure où on les considère par définition comme uniques et non reproductibles. Ainsi, les centres historiques peuvent prétendre constituer la source de rentes de monopole car il s'agit du type même d'espace urbain perçu positivement comme unique à l'heure de la "patrimoniaphilie ambiante » (Gravari-Barbas et Guichard-Anguis, 2003). Comme le souligne G. Waitt, « urban heritage provides a particular mechanism for cities to specialize by calling upon their unique and distinctive past events » $(2000$, p. 840). Cela est particulièrement vrai pour un type d'acteur urbain, les commerçants.

22 Si la tendance la plus visible et la plus médiatisée reflétant ce besoin croissant de différenciation des points de vente s'exprime d'abord dans le milieu du luxe par des productions architecturales spectaculaires, atypiques et emblématiques conçues par la crème des "starchitectes » (Crewe, 2010), le patrimoine peut également être mis au service de cette stratégie de différenciation. Dans le cas du Marais, l'investissement de l'ancien hammam de la rue des Rosiers par la marque COS («Collection Of Styles», 
version haut de gamme de la chaîne suédoise $H \& M$ ) s'explique certes par l'importance de la surface disponible $\left(450 \mathrm{~m}^{2}\right.$ - rapidement doublés - surface très rare dans ce quartier où les locaux commerciaux sont généralement de très petite taille) ainsi que par le récent succès commercial de cette rue. Cependant, les caractéristiques architecturales de ce bâtiment - datant selon la commission du Vieux Paris de la fin du xixe siècle - au revêtement de granito rouge typique des années 1920, avec une enseigne toujours très visible révélatrice de l'ancienne fonction des lieux (« Hammam Saint-Paul »; "Piscine ») et des sculptures en façade, ainsi que son passé de haut-lieu de la sociabilité juive "traditionnelle », ont probablement aussi joué un rôle dans le choix de ce local.

Figure 5 : Le premier COS de France dans l'ancien hammam Saint-Paul rue des Rosiers (Marais), une stratégie de différenciation?

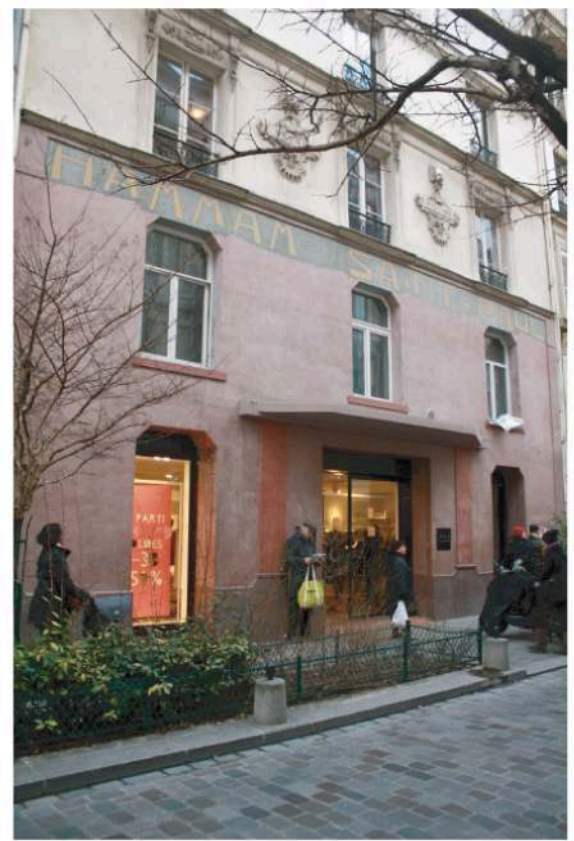

"Inutile de chercher les lettres rouges de la firme suédoise au 4 rue des Rosiers. Si c'est bien elle qui a fait main basse sur I'ancien hammam, c'est, pour la première fois en France, sous le nom de $\operatorname{COS}$ qu'elle a ouvert un nouveau magasin. $\operatorname{COS}$ pour Collection of Style. Car la nouvelle boutique du Marais se veut plus haut de gamme que les autres de la capitale. Les lettres noires de l'enseigne, qui se détachent sur la façade vieux rose, collent parfaitement avec l'esprit du bon chic du quartier $[\ldots]$...

Le Parisien, 17 mars 2009

"Autre de ces bonnes adresses parisiennes peu coûteuses: $\operatorname{COS}$ qui s'est établie en mars 2009 à la rue des Rosiers. COS, c'est l'abréviation de Collection of style, la ligne indépendante et plus haut de gamme - donc plus chère - de $\mathrm{H}$ \& $M$. On ne la trouve que dans quelques grandes métropoles pour I'instant. Le magasin a pris ses quartiers dans un bâtiment à la façade classée, l'ancien hammam de cette rue, centre de la communauté juive ashkénaze du Marais. Là, pas d'architecture d'intérieur signée, mais un jeu assez amusant pour les connaisseurs".

Le Temps, 16 octobre 2010

Source de la photo : A.-C. Mermet (janvier 2011)

Le choix d'un bâtiment à forte connotation patrimoniale a dans ce cas participé d'une stratégie de différenciation que deux indices permettent de déceler. D'une part, la façade n'a absolument pas été retouchée (alors que l'immeuble ne fait l'objet d'aucune protection sur le PSMV ${ }^{10}$, il s'agit d'un « immeuble non protégé pouvant être remplacé ou amélioré ", contrairement à ce qu'on peut lire dans de nombreux articles de presse). La marque a même choisi de ne pas faire figurer d'enseigne visible sur la devanture (hormis une sobre et discrète plaque noire à droite de la porte d'entrée). En outre, la revue de presse qui a été constituée au sujet de cette boutique montre que c'est d'abord par l'originalité de sa façade que l'on parle de ce point de vente. Choisir un écrin patrimonial, c'est donc aussi bénéficier d'un support permettant de faire parler de soi, de « faire le buzz » grâce à l'originalité architecturale de cette enveloppe. On peut donc légitimement penser que le choix de ce local correspond à une logique d'exploitation d'un « capital symbolique collectif » (Harvey, 2008, p. 45) porté par le bâti patrimonial et rentabilisé par la marque. 
24 Mais cette mise en résonnance ne se limite pas à la question de l'unicité. Dans l'utilisation de la «starchitecture » comme dans celle du patrimoine, il s'agit également de raconter une histoire au consommateur, d'inscrire la fréquentation de la boutique dans un univers plus riche que celui de la simple fonctionnalité marchande. Dans le cas du patrimoine, les entretiens font émerger une autre forme de synergie entre ce cadre patrimonial et la boutique fondée sur l'idée d'une forme de continuité entre le passé des murs et du décor et le présent de la boutique.

\section{B. Continuité}

L'utilisation du cadre patrimonial permet également d'inscrire le point de vente non pas dans l'immédiateté de l'acte d'achat mais dans le temps long des vieilles pierres. Le choix de s'installer dans un centre historique est en effet motivé dans de nombreux cas par la volonté exprimée par les commerçants de s'inscrire dans la continuité avec les formes plus anciennes - plus «traditionnelles »-du commerce. Il s'agit ici pour ces acteurs de profiter de la fonction légitimante du patrimoine (Veschambre, 2008, p. 65), à l'échelle du quartier comme à celle du local commercial.

Le choix d'une localisation en centre historique, indépendamment des caractéristiques architecturales du local commercial, apparaît dans les discours des commerçants comme un premier élément suggérant une forme de continuité avec les activités «traditionnelles » de ces centres historiques. Ainsi, la créatrice de la marque Genna M., qui a récemment ouvert une boutique indépendante dans laquelle elle diffuse les robes qu'elle crée et confectionne elle-même, rue des 3 Molettes dans le Vieux Lille, explique avoir délibérément choisi de s'installer dans ce quartier parce qu'elle inscrit son activité en véritable continuité avec l'artisanat ancien, et notamment avec la tradition de la confection de la capitale de cette ancienne grande région textile (on y trouvait encore en 1965 de nombreux ateliers de confection, des bonnetiers, des fourreurs etc. ${ }^{11)}$. On pourrait également citer le discours de la propriétaire d'une boutique de décoration/brocante dans la rue Bartholomé Masurel du Vieux Lille qui explique : « Il y avait une volonté délibérée de ma part d'être dans un quartier ancien. Je n'aurais pas envisagé ouvrir ma boutique dans un centre commercial, ou dans une rue... [...] J'ai naturellement... j'ai même pas cherché ailleurs, c'était une évidence. Je pensais que mon type de commerce avait sa place dans une partie ancienne de la ville. Et en centreville, pas dans une banlieue ». Le caractère ancien des produits vendus est ici clairement mis en résonnance avec le caractère ancien du quartier investi en s'inscrivant dans une forme de continuité avec un artisanat plus « traditionnel ».

Mais c'est à l'échelle de la boutique que cette stratégie de mise en résonnance symbolique entre les caractéristiques patrimoniales de ces lieux et celles véhiculées par les boutiques et les produits vendus est la plus facilement lisible, comme l'illustre le cas de la boutique indépendante Senteur de fée, située rue de Sévigné, dans la partie sud du Marais. La fondatrice de cette marque de produits cosmétiques qu'elle crée dans un atelier situé à Montreuil et qu'elle diffuse dans cette boutique, explique ainsi avoir choisi ce type de mobilier afin de "prendre le relais » d'un commerce "traditionnel » en voie de disparition dans le quartier. La "grande table de bois, [le] grand comptoir, comme il y en avait avant, dans l'ancien temps " autour desquels s'articule l'aménagement de la boutique n'ont pas seulement une fonction esthétique, mais aussi une fonction mémorielle, destinée à établir matériellement, par la décoration «à 
l'ancienne ", avec un mobilier "authentiquement» ancien, une forme de continuité symbolique avec la vie commerçante passée du quartier que cette commerçante a par ailleurs évoqué avec nostalgie dans l'entretien.

On peut également interpréter la forte concentration de commerces se revendiquant de « créateurs » dans le haut-Marais comme l'affirmation d'une forme de continuité entre le passé artisanal du quartier et l'identité créative que l'on veut associer à ces boutiques. Ainsi, plusieurs créateurs ou responsables de boutiques de créateur du Marais ont pu expliquer que le passé artisanal du quartier a constitué une des raisons qui a motivé le choix d'une implantation dans ce quartier (Mermet, 2013).

Les valeurs d'ancienneté portées par la présence du cadre patrimonial constituent donc un support privilégié pour les commerçants (qu'ils soient créateurs indépendants ou chaînes internationales) pour inscrire leur boutique et leur activité dans un temps long fictif, légitimant ainsi leur présence dans l'espace urbain et leur permettant de lier leur activité à des formes "ancestrales » de commerce à l'heure du plus contemporain « marketing de la nostalgie » (Divard et Robert-Demontrond, 1997).

\section{Intimité et proximité}

Autre valeur qui fonde cette synergie entre la dimension patrimoniale des lieux investis et les produits proposés à la vente, la notion d'intimité est également centrale dans les discours des commerçants. L'une des grandes tendances du merchandising contemporain cherche en effet à reconstituer des " espaces confidentiels » (Sackrider et al., 2008, p. 107-108). Ainsi, à l'échelle du quartier comme à celle de la boutique, s'installer en centre historique peut constituer une stratégie de choix pour qui veut adopter ce type de merchandising. La responsable de la boutique Sessùn dans le Vieux Lille précédemment évoquée (cf. figure 3c) explique ainsi que chaque boutique de la marque est aménagée de façon à recréer " un esprit cosy, assez chaleureux » destiné à faire en sorte que les clients se sentent bien dans la boutique :

- Et quand vous avez pensé l'aménagement intérieur, vous avez voulu véhiculer quoi comme idée?

- Ben en fait le but du jeu, c'est que, nous, comme les clientes, elles se sentent chez elles. Donc on voulait vraiment quelque chose de chaleureux, d'où la brique apparente, le parquet qui est resté un peu brut, un peu...

- Il était déjà là le parquet?

- Non non, ça on l'a mis parce qu'avant... Donc le mur de brique était complètement couvert de placo, donc tout était blanc partout. Et le sol c'était le même revêtement que l'escalier en fait. Une espèce de béton peint... donc totalement différent. Très très moderne, donc un peu plus froid au niveau ambiance, mais bon [...]. Et tous les meubles ont été fabriqués par des architectes.

\section{- Ils sont spécifiques à cette boutique-là?}

- Oui, tout à fait. Vous retrouverez tout ce qui est les caissons de bois par exemple, même les meubles, tout a vraiment été acheté pour la boutique et tous les petits miroirs dans l'escalier ont été chinés par exemple, le petit fauteuil en rotin là-bas pareil, les petits tableaux qui sont en cabine, c'est pareil. Tout est vraiment chiné, pour que tout soit vraiment dans le même esprit. Et pour que ce soit le plus cosy possible. C'est surtout ça notre but. Qu'on se sente bien, et que ce soit chaleureux. Après, c'est les inconvénients aussi, c'est 
qu'au niveau lumière aussi, on est sur des lumières tamisées etc., donc des fois les couleurs sont pas mises en avant comme elles devraient l'être mais bon, après, c'est un parti pris.

31 Le recours déjà évoqué à l'usage de mobilier chiné est ici explicitement destiné à suggérer à la clientèle des valeurs, des idées, des émotions dont la marque veut se prévaloir : le côté chaleureux, intimiste et « comme à la maison ».

32 À plus petite échelle, comme pour la notion de continuité, c'est aussi par le choix d'un quartier historique et non seulement d'un local à forte connotation patrimoniale, que passe cette construction symbolique avec la notion d'intimité. Ainsi, la principale raison qui pousse la marque Chattawak précédemment évoquée à choisir des implantations en centres historiques réside dans la volonté de création d'une relation de " proximité » avec la clientèle, que l'on peut rattacher à cette notion d'intimité :

- [Les centres-villes] véhiculent une ambiance particulière, davantage de... d'échange justement. Parce qu'on est une enseigne, on est dans le prêt-à-porter féminin, et je pense qu'on veut retrouver un petit peu ce côté petit commerçant de proximité. Et ce côté commerçant de proximité, on veut vraiment le garder chez Chattawak, c'est important [...]. On a ouvert par exemple aux Abesses, c'est notre dernière boutique qui a ouvert, donc là on est complètement dans cet esprit petit village et boutique de quartier. Voilà, je pense que ça correspond à tout ce que j'ai pu dire avant sur l'état d'esprit de Chattawak en général, ce qu'on veut véhiculer, l'échange que l'on veut avoir avec les clientes, le centre-ville correspond mieux $\grave{a} c ̧ a[\ldots .$.$] .$

On observe donc une association symbolique très nette entre la localisation en centreville ou en centre historique et cette idée de " commerce de proximité », en lien avec la notion de village, de quartier, d'esprit d'interconnaissance. C'est donc d'abord parce que la matérialité du centre historique - avec pour toile de fond le décor patrimonial est symboliquement associée à l'idée de village et de proximité que la marque privilégie ce type d'implantation

\section{Le patrimoine au service de la production d'un espace de consommation haut de gamme}

In fine, la richesse des correspondances symboliques permises par ce support patrimonial dont on met en exergue les signes dans ces boutiques, participe de la production d'un espace de consommation haut de gamme et prestigieux.

De fait, les structures commerciales du Marais comme du Vieux Lille ont connu depuis les années 1990 une forte montée en gamme. Un recensement des commerces en 2011 a permis de montrer que $87 \%$ des commerces du Vieux Lille relevaient de la catégorie «moyen-haut de gamme », ce qui n'était pas le cas avant les années 1990. 
Figure 6 : Niveau de gamme des commerces du Vieux Lille

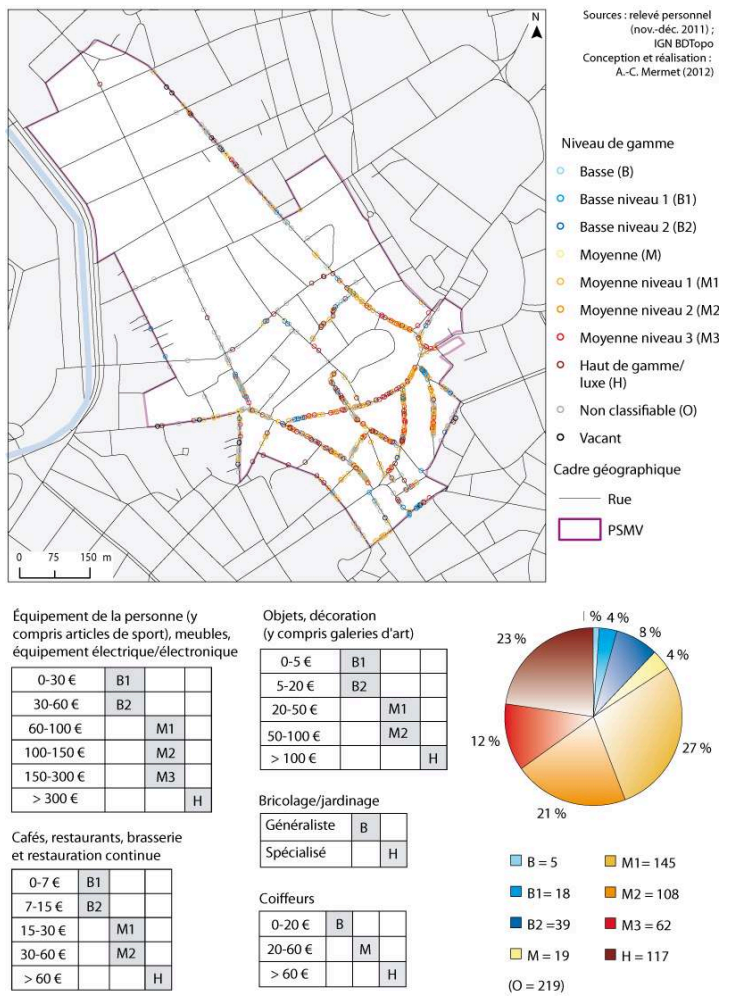

Cette carte fait clairement apparaître une dominante des commerces de moyen-haut de gamme dans le périmètre du secteur sauvegardé ( $87 \%$ des commerces au total). La partie sud de la Grand Place (rue des Manneliers) annonce le plateau piétonnier à dominante davantage mass market. Cette carte est à comparer avec celle établie par N. Lebrun en 2002 à partir d'un indice de gamme à l'îlot pour l'ensemble Vieux Lille/ plateau piétonnier (Lebrun, 2002, p. 253) qui montrait déjà la spécialisation du Vieux Lille vers le " très haut de gamme ». Le plateau piétonnier apparaissait sur cette carte soit comme " milieu de gamme ", soit comme "banal et sans image ». Cette tendance s'est confirmée voire renforcée en dix ans.

S'il ne s'agit pas du seul élément explicatif permettant de comprendre cette montée en gamme, la présence d'un cadre patrimonial dont la réhabilitation a permis d'en marquer très visiblement les signes, peut partiellement expliquer ce phénomène. Jean Baudrillard notait déjà le "statut particulier de l'objet ancien " auquel il accorde un chapitre dans son Système des objets $(1968$, p. 105) et dont il souligne dans sa théorie sur La société de consommation « le prestige très particulier » (1970, p. 78). De fait, les logiques des commerçants, et particulièrement des enseignes moyen-haut de gamme, privilégient explicitement des localisations dans des contextes urbains aux connotations sociales prestigieuses, entrant en résonnance avec leur standing, comme cet extrait d'entretien avec le directeur général de la marque de chaussures Stéphane Gontard :

- Est-ce que c'est vraiment une volonté de vous mettre en centre-ville, ou est-ce que c'est un hasard?

- Alors... il y a deux raisons principales. C'est souvent... la clientèle de centre-ville a un pouvoir d'achat... enfin ceux qui viennent au centre-ville ont un pouvoir d'achat qui est probablement supérieur à ce qui se fait en périphérie [...]. Et quant à ouvrir... on 
pourrait aussi penser ouvrir des magasins de périphérie dans ce qu'on appelle des boîtes à vendre. Et... sans parler de l'investissement qui est souvent important, mais notre produit correspond pas à ce genre de distribution si vous voulez...

- Comment ça?

- C'est-à-dire, nous on a un produit moyen-haut de gamme, et, en périphérie, qui ont des locaux style Halle aux chaussures, Gémo, etc., c'est plus grande distribution si vous voulez. Nous on a un produit qui est nettement au-dessus de ça, donc ça correspondrait pas. Oui, c'est un type de distribution qui correspond pas à notre produit.

Il y a donc implicitement dans ce discours l'idée que le cadre esthétique extérieur dans lequel s'inscrit son commerce doit correspondre à un certain niveau de gamme: le commerce bas de gamme serait à sa place dans les boittes (Péron, 1993, 2004) des zones commerciales périphériques alors que ses produits plutôt haut de gamme seraient davantage valorisés dans un quartier central mais aussi patrimonial. On pourrait également évoquer le cas de la marque de prêt-à-porter féminin moyen-haut de gamme Ekyog, en plein développement, qui choisit spécifiquement des emplacements en cohérence avec son positionnement de gamme : par exemple, la boutique du Marais, rue des Francs-Bourgeois, se situe dans une ancienne boulangerie inscrite à l'inventaire supplémentaire des monuments historique et ne constitue pas un cas isolé. La plusvalue symbolique portée par ce cadre hautement patrimonial semble ici compenser clairement les surcoûts induits par ce type de localisation très contrainte.

La mise en articulation entre les signes du commerce et les signes du patrimoine ne se limite donc pas à une juxtaposition de ces signes, mais crée un véritable discours visuel né de cette articulation.

\section{Conclusion :}

Le cadre patrimonial apparaît donc comme une réelle plus-value environnementale aux yeux des commerçants installés en centre historique. Sur le plan esthétique tout d'abord, les spécificités architecturales de ces quartiers, marquées par l'omniprésence de « signes du patrimoine » rendus particulièrement visibles par les lourdes opérations de réhabilitation qui ont suivi la mise en place des secteurs sauvegardés, constituent un support particulièrement prisé pour produire des espaces commerciaux hautement qualitatifs, en phase avec l'affirmation contemporaine diffuse d'un goût pour les vieilles pierres. Sur le plan symbolique ensuite, la localisation dans un contexte patrimonial - envisagé à l'échelle du quartier comme un environnement global ou à l'échelle de la boutique comme un environnement immédiat - permet une réelle mise en résonnance entre ces valeurs symboliques effectivement attachées au cadre patrimonial et les valeurs désirées par les boutiques, et peut donc se lire en termes sémiologiques. On aboutit donc à la production d'un véritable discours visuel dont les différentes composantes participent à l'affirmation des centres historiques comme des espaces de consommation plutôt haut de gamme et prestigieux, en tout cas distinctifs. Mais cette plus-value n'est pas que symbolique. In fine, l'objectif est bien la valorisation économique de ce capital patrimonial via ces registres esthétiques et symboliques en attirant une clientèle à haut pouvoir d'achat, particulièrement sensible à ce type d'environnement urbain (gentrifieurs, touristes...). 


\section{BIBLIOGRAPHY}

Barthes R., 1967, Système de la mode, Paris, Seuil, 326 p.

Baudrillard J. 1970, La Société de consommation : ses mythes et ses structures, Paris, Gallimard, 1970, $318 \mathrm{p}$.

Baudrillard J., 1968, Le système des objets, Paris, Gallimard, 245 p.

Boudon P., 1977, « Sémiotique de l'espace », Communication, n 27, p. 1-12.

Bourdieu P., 1979, La distinction : critique sociale du jugement, Paris, Éditions de Minuit, VIII-670 p.

Deleuze G., 1972, « À quoi reconnaît-on le structuralisme ? », in Histoire de la philosophie. Le XXe siècle, Hachette, Paris, vol.8, p. 299-335.

Dioux J. et Dupuis M., 2009, La distribution : stratégies des réseaux et management des enseignes, Paris, Pearson Education, $481 \mathrm{p}$.

Divard R. et Robert-Demontrond P., 1997, « La nostalgie : un thème récent dans la recherche marketing ", Recherche et Applications en Marketing, vol. 12, nº 4, p. 41-62.

Featherstone M., 1991, Consumer culture and postmodernism, London; Newbury Park (Calif.); New Delhi, Sage, 164 p.

Fitoussi B., Fauconnet F. et Leopold K., 1997, Les boutiques à Paris : vitrines d'architectures, Paris, Picard, $221 \mathrm{p}$.

Goss J., 1993, "The " Magic of the Mall »: An Analysis of Form, Function, and Meaning in the Contemporary Retail Built Environment ", Annals of the Association of American Geographers, vol. 83, $\mathrm{n}^{\circ} 1$, p. $18-47$.

Gravari-Barbas M. et Guichard-Anguis S. (dir.), 2003, Regards croisés sur le patrimoine dans le monde à l'aube du XXI siècle, Paris, Presses Paris Sorbonne, 964 p.

Harvey D., 2008, Géographie de la domination, Paris, Les prairies ordinaires, 118 p.

Lamizet B., 2007, « La polyphonie urbaine : essai de définition », Communication et organisation, $\mathrm{n}^{\circ}$ 32, p. $14-25$.

Lash S. et Urry J., 1994, Economies of signs and space, London, Sage Publications, $360 \mathrm{p}$.

Lebrun N., 2002, Centralités urbaines et concentrations de commerces, Thèse de $3^{\mathrm{e}}$ cycle de géographie, Université de Reims Champagne-Ardennes, Reims, 2002, 511 p.

Lefebvre H., 2000 [1974], La production de l'espace, Paris, Anthropos, 485 p.

Lemoine R., 2006, Transformation et réhabilitation de l'habitat traditionnel lillois en magasin, École Centrale de Lille.

Mermet A.-C., 2013, « Le Marais, quartier créatif ou vitrine de créateurs ? », Bulletin de l'association des géographes français, vol. 90, $\mathrm{n}^{\circ}$ 2, p. 186-201.

Miller D. (dir.), 1995, Acknowledging consumption: a review of new studies, London, Routledge, 341 p.

Péron R., 2004, Les boîtes : les grandes surfaces dans la ville, Nantes, L'Atalante, 221 p.

Péron R., 1993, La fin des vitrines : des temples de la consommation aux usines à vendre, Cachan, Éditions de l'École Normale Supérieure de Cachan, 306 p. 
Rieunier S., 2009, Le marketing sensoriel du point de vente: créer et gérer l'ambiance des lieux commerciaux, Paris, Dunod, 294 p.

Sackrider F., Guidé G. et Hervé D., 2008, Lèche-vitrines : Distribution et merchandising visuel dans la mode, $2^{\mathrm{e}}$ édition, Paris, Éditions du Regard, $232 \mathrm{p}$.

Veschambre V., 2008, Traces et mémoires urbaines : enjeux sociaux de la patrimonialisation et de la démolition, Rennes, Presses universitaires de Rennes, $315 \mathrm{p}$.

Waitt G., 2000, « Consuming heritage: Perceived historical authenticity », Annals of Tourism Research, vol. 27, $\mathrm{n}^{\circ} 4$, p. $835-862$.

Zukin S., 2010, Naked city: the death and life of authentic urban places, Oxford, New York, Auckland, Oxford University Press, $294 \mathrm{p}$.

\section{NOTES}

1. Conduite dans le cadre d'une thèse soutenue en décembre 2012.

2. Dans les périmètres délimités par les secteurs sauvegardés (tous les commerçants enquêtés dans le Marais et le Vieux Lille sont localisés dans ce périmètre).

3. Même si la distinction de statut est parfois subtile dans le cas de marques émergentes ayant démarré dans le quartier.

4. Défini comme le «mode de présentation d'un produit » (Sackrider et al., 2008, p. 13), ce qui englobe la localisation du point de vente, son aménagement, son assortiment, l'attitude du personnel vis-à-vis de la clientèle, etc., c'est-à-dire tout ce qui va avoir une influence sur la perception par le client, du produit vendu.

5. Succursale.

6. Indépendant.

7. Service Territorial de l'Architecture et du Patrimoine, au sein duquel exercent les architectes des bâtiments de France.

8. Hors corner en grand magasin (données de 2012).

9. Aujourd'hui déplacée rue Vieile-du-Temple.

10. Plan de sauvegarde et de mise en valeur, document d'urbanisme lié au secteur sauvegardé et se substituant au Plan local d'urbanisme.

11. Source: Annuaire Ravet-Anceau, 1965.

\section{ABSTRACTS}

French historic centres gained a real heritage function during the last century, highlighting both the material and symbolic values of these kind of neighbourhoods, with important consequences on their contemporary transformations. This paper analyses more particularly interactions between the heritage and retail functions, focusing on the discourses of retailers. It aims to understand how retailers exploit heritage as a real aesthetic and symbolic capital to produce a qualitative space of consumption. 
Les centres historiques français ont acquis durant le siècle dernier une indéniable fonction patrimoniale, valorisant sur le plan matériel mais aussi symbolique ces portions d'espaces urbains, ce qui n'est pas sans conséquences sur leurs transformations contemporaines. Cet article analyse plus particulièrement les interactions entre cette fonction patrimoniale et la fonction commerciale qui tend à se réaffirmer dans ces espaces centraux, en mettant l'accent sur un type d'acteur particulier, les commerçants. Il s'agit de montrer, à travers une analyse de discours, que les commerçants utilisent ce cadre patrimonial comme un véritable capital esthétique mais aussi symbolique qu'ils exploitent pour produire un espace de consommation à la fois qualitatif et vecteur de valeurs positives, associées aux boutiques et aux produits vendus.

Seit dem letzten Jahrhundert haben französische historische Stadtzentren eine wirkliche Erbfunktion erhalten, die ihren materiellen und symbolischen Wert betont hat, und sich auf die aktuellen Veränderungen dieser Stadtviertel stark auswirkt. Dieser Artikel untersucht die Wechselwirkungen zwischen Erbe und Handelsfunktion insbesondere durch eine Analyse der Tätigkeit der Händler. Er zeigt, wie Händler das Erbe als ästhetisches und symbolisches Kapital benutzen, um einen positiv konnotierten Verbrauchsraum zu produzieren.

INDEX

Keywords: heritage, historic centre, Marais, retail, spatial semiology, symbolic capital, Vieux Lille

Schlüsselwörter: Erbe, Handel, Historisch Zentrum, Marais, Raumliche Semiotik, Symbolisch Kapital, Vieux Lille

Mots-clés: capital symbolique, centre historique, commerce, Marais, patrimoine, sémiologie spatiale, Vieux Lille

\section{AUTHOR}

ANNE-CÉCILE MERMET

ATER à l'ENS de Lyon - EA-EIREST, Université Paris 1 Panthéon-Sorbonne 